Proceedings

\title{
Tailoring the Electrochemical and Morphological Properties of Electropolymerized and Dropcast rGO:PSS-PEDOT:PSS Transducers for Ion-Selective Sensors ${ }^{+}$
}

\author{
Ihda Uswatun Shalihah Shohibuddin and Wan Wardatul Amani Wan Salim * \\ Department of Biotechnology Engineering, Faculty of Engineering, International Islamic University Malaysia, \\ Gombak, Kuala Lumpur 50728, Malaysia; \\ * Correspondence: asalim@iium.edu.my \\ + Presented at the 1st International Electronic Conference on Biosensors, 2-17 November 2020; Available \\ online: https://iecb2020.sciforum.net/.
}

Received: date; Accepted: date; Published: date

\begin{abstract}
Fabrication of ion-selective sensors for continuous measurement in fluids depends on understanding the electrochemical and morphological properties of transducers. Electropolymerized nanomaterials essentially offer stable transducers that can reduce measurement drifts. This study aims to elucidate the electrochemical and morphological characteristics of electropolymerized reduced graphene oxide stabilized in polystyrenesulfonate and poly(3,4ethylenedioxythiophene): polystyrenesulfonate composites on screen-printed carbon electrodes (rGO:PSS-PEDOT:PSS/SPCEs) using scanning electron microscopy (SEM) and cyclic voltammetry (CV) in $0.1 \mathrm{M}$ ferricyanide (Fe(CN)64/3-). We fabricated the rGO:PSS-PEDOT:PSS/SPCEs by two different techniques: electropolymerization deposition (EPD) and drop-casting (DC). Results revealed smaller peak-to-peak potential separation $(\triangle \mathrm{Ep})$ of $360 \mathrm{mV}$ for EPD rGO:PSSPEDOT:PSS/SPCEs, compared to $510 \mathrm{mV}$ for the DC rGO:PSS-PEDOT:PSS/SPCEs. A smaller $\triangle E p$ indicates higher reversibility and faster electron-transfer rate at the electrode-analyte interface. SEM results showed EPD rGO:PSS-PEDOT:PSS/SPCEs have the roughest surface among electrodes; homogeneous globular structures with diameter range of 1.4-5.3 $\mu \mathrm{m}$ covered the electrode surface. In terms of electrode integrity in fluids, cracks can be seen on the surface of DC PEDOT:PSS/SPCEs after undergoing $\mathrm{CV}$ in $0.1 \mathrm{M} \mathrm{Fe}(\mathrm{CN}) 64 / 3-$, whereas rGO:PSS-PEDOT:PSS/SPCEs for both deposition methods maintained their integrity. Globular structures of rGO:PSS-PEDOT:PSS using EPD methods remained after undergoing CV. The results suggest that EPD serves as a potential method to fabricate a stable transducer for ion-selective sensing. This study aims to elucidate performance of nanocomposites via EPD methods, to develop stable ion-selective sensors for physiological and environmental applications.
\end{abstract}

Keywords: electropolymerization; drop-cast; reduced graphene oxide; PEDOT:PSS; rGO:PSS; allsolid-state ion-selective sensors

\section{Introduction}

Ion-selective sensors have been an attractive choice of sensing technology for clinical, food, and environmental applications owing to sensitive, low-cost, and straightforward fabrication processes, while offering in addition the ability to monitor analytes in fluids [1-6]. Driven by advancements in nanotechnology, modern ion-selective sensors have evolved from conventional liquid-filled ionselective electrodes (ISEs) into all-solid-state ISEs (AS-ISEs) that are easier to miniaturize and integrate into sensing platforms [7,8]. However, prolonged use of AS-ISEs in fluids can affect the 
integrity of sensors and result in measurement drifts. Continuous real-time monitoring is required for various applications encompassing water-quality monitoring [9-11], in-vivo measurement for physiological processes [12-15], and nutrient monitoring in food [16]. Yet, conventional ISEs with inner filling solution are sensitive to evaporation and osmotic pressure, resulting in liquid volume change and hence frequent calibration [17]. Furthermore, handling of solutions requires microfluidics and automation. In this regard, there is a need to develop a robust and stable sensor that can function continuously in fluids with minimal measurement drift and less need for calibration during use. Such minimal drift with high potential stability of AS-ISEs can be achieved using solid-contact materials $[18,19]$.

Previous work reported several techniques to deposit solid-contact materials, such as dropcasting (DC) [20,21], spin coating [22,23], inkjet printing [24-26], and electropolymerization deposition (EPD) [27,28]. DC is the simplest, consisting of two easy steps: casting the materials onto the WEs followed by drying the electrodes. However, transducer materials deposited by drop-casting are relatively thick, lack homogeneity along the WEs, and possess weak adhesion to electrode surfaces. As a result, electrodes modified via drop-casting are not mechanically robust; measurements results can vary between electrodes, so the technique is unsuitable for scaling-up purposes. EPD, however, is a promising method to fabricate robust and stable AS-ISEs owing to stronger molecular interaction between the polymers and the electrode surface, homogeneous thinfilm deposition whose thickness can be controlled by changing the number of potential sweep cycles, and the capability to coat nanosize WEs [29]. Electropolymerization of 3,4-ethylene dioxythiophene (EDOT) in poly(sodium-4-styrenesulfonate) (NaPSS) and lithium perchlorate solution $\left(\mathrm{LiClO}_{4}\right)$ occurs when electrodes oxidize the EDOT monomers, making the resulting cations combine with themselves, thus producing higher oligomers of electropolymerized PEDOT:PSS [30]. The electropolymerized PEDOT:PSS has been tested for stability of the charge/discharge cycle using cyclic voltammetry (CV) and for its adhesion to electrode surfaces in flowing aqueous media [31].

Besides electropolymerized PEDOT:PSS, several studies have employed electropolymerization of graphene-based nanomaterials and PEDOT as a composite. PEDOT was electropolymerized from an aqueous solution with graphene oxide (GO) as the only charge-compensating counterion. During electropolymerization, GO can be incorporated into the positively charged PEDOT matrix because of the polar hydroxyl and epoxy groups, thus making the GO surface negatively charged. At present, the electropolymerization of rGO-PEDOT:PSS composite has been done with two-step processes, either by doing electropolymerization on the rGO surface [32], or by reducing GO to rGO electrochemically on top of the electropolymerized PEDOT layer [33].

In this study, we employed a one-step electropolymerization of EDOT in a complex electrolyte solution with an aqueous dispersion of reduced graphene oxide. The electropolymerized reduced graphene oxide stabilized in polystyrenesulfonate and poly(3,4-ethylenedioxythiophene): polystyrenesulfonate composite on screen-printed carbon electrodes (rGO:PSS-PEDOT:PSS/SPCEs) was then electrochemically characterized by cyclic voltammetry measurement. We used reduced graphene oxide stabilized in polystyrenesulfonate (rGO:PSS) to avoid rGO stacking while promoting hydrophilicity of the nanomaterial, allowing rGO:PSS to be dissolved in aqueous solution. In addition to electrochemical characterization, we also performed surface-morphology characterization to investigate the effect of fluids on electrode-surface morphology after CV measurement. A schematic of the experimental setup is illustrated in Figure 1. This study essentially offers a new platform to develop robust and stable AS-ISEs, which are particularly useful for monitoring ion concentrations continuously and in real-time for physiological and environmental applications. 
a

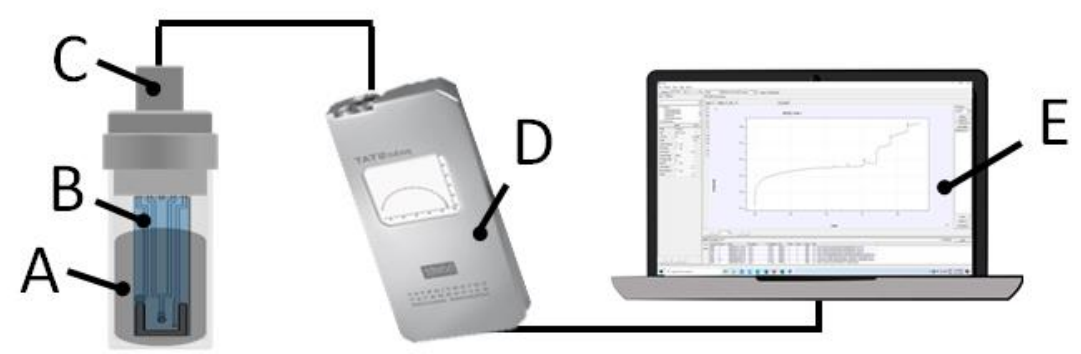

b
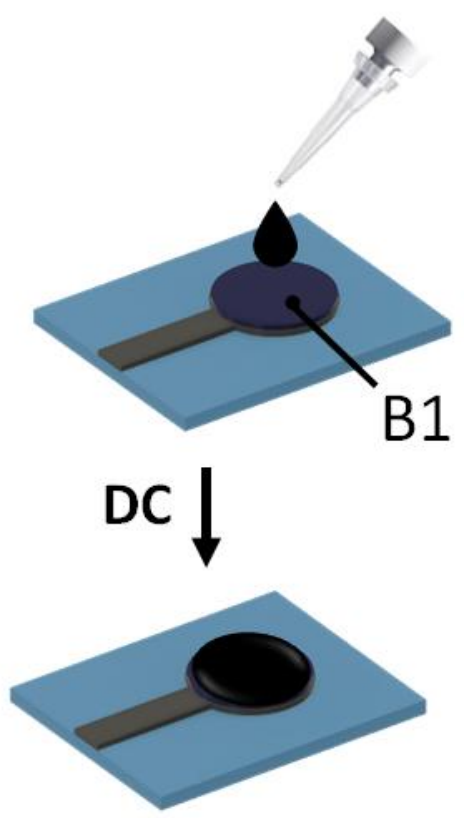

C

- rGO:PSS • EDOT

- $\mathrm{LiClO}_{4}$ - PSS

- rGO:PSS-PEDOT:PSS
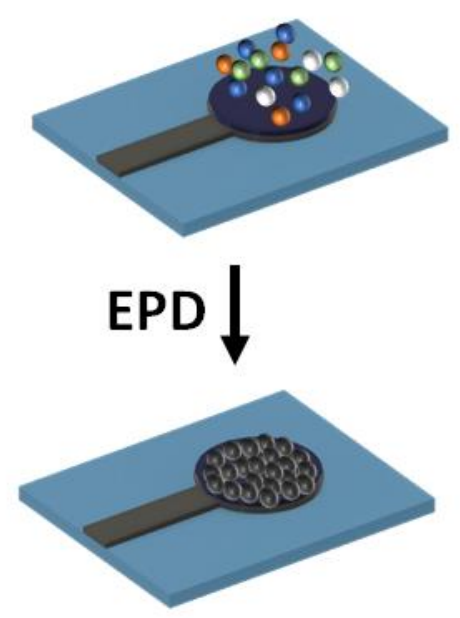

\section{rGO:PSS-PEDOT:PSS/SPCES rGO:PSS-PEDOT:PSS/SPCES}

Figure 1. All-solid-state ion-selective sensors. (a) Schematic of all-solid-state ion-selective sensors for continuous monitoring in fluids. A: sample fluid, B: screen-printed carbon electrode (SPCE), C: connector between electrode and pocketSTAT ${ }^{\mathrm{TM}}$, D: pocketSTAT ${ }^{\mathrm{TM}}$, E: IVIUM software. Fabrication steps of rGO:PSS-PEDOT:PSS/SPCEs by (b) drop-casting (DC) and (c) electropolymerization deposition (EPD) techniques. B1: expanded view of SPCEs showing the working electrode (WE).

\section{Materials and Methods}

\subsection{Materials and Instrument}

Screen-printed carbon electrodes (SPCEs, RRPE1001C) with diameter $\varnothing=2 \mathrm{~mm}$ were purchased from Pine Instrument Company, Grove City, PA, USA. A PocketSTAT ${ }^{\mathrm{TM}}$ was purchased from IVIUM Technologies, Eindhoven, the Netherlands. Reduced graphene oxide stabilized with poly(sodium 4styrenesulfonate) (rGO:PSS, 900197), poly(3,4-ethylenedioxythiophene):poly(styrenesulfonate) (PEDOT:PSS, 483095), $\mathrm{LiClO}_{4}$, EDOT, and NaPSS were purchased from Sigma-Aldrich, St. Louis, MO, USA. 98\% concentrated sulfuric acid (H2SO4, 1407-80), and potassium ferricyanide ( $\mathrm{K}_{3} \mathrm{Fe}(\mathrm{CN}){ }_{6}$, 696900) were purchased from R\&M Chemicals, Selangor, Malaysia. Analytical-grade chloride salts of potassium and sodium were procured from Sigma-Aldrich, St. Louis, MO, USA. All chemicals of analytical grade were used as received without further purifications. Deionized water (DI) was used throughout the experiment. 


\subsection{Fabrication of Drop-Cast and Electropolymerized rGO:PSS-PEDOT:PSS/SPCES}

SPCEs were activated via cyclic voltammetry for 3 cycles in $0.1 \mathrm{~N} \mathrm{H}_{2} \mathrm{SO}_{4}$ at potentials of $2.5 \mathrm{~V}$ to $-2.5 \mathrm{~V}$ and scan rate of $100 \mathrm{mV} / \mathrm{s}$. The activated SPCEs were rinsed in DI water and left to dry. To prepare rGO:PSS-PEDOT:PSS/SPCEs, two techniques were used in modifying the working electrode: drop-casting (DC) and electropolymerization deposition (EPD). For DC, rGO:PSS was mixed with PEDOT:PSS to prepare a rGO:PSS-PEDOT:PSS composite. To fabricate rGO:PSS-PEDOT:PSS/SPCEs, $3 \mu \mathrm{l}$ rG:PSS-PEDOT:PSS was drop-cast onto the WE of an SPCE. For EPD, the NaPSS, EDOT, and rGO:PSS were dissolved in $0.1 \mathrm{M} \mathrm{LiClO}_{4}$. The galvanostatic technique by applying constant current was used to electropolymerize EDOT and rGO:PSS to rGO:PSS-PEDOT:PSS. Both DC and EPD rGO:PSS-PEDOT:PSS/SPCEs were left to dry for $24 \mathrm{~h}$ under a laminar flow hood (Esco Micro, ADC4C1, Shah Alam, Selangor, Malaysia).

\subsection{Cyclic Voltammetry of $r G O: P S S-P E D O T: P S S / S P C E S$}

Cyclic voltammetry (CV) was performed for unmodified SPCEs, DC rGO:PSSPEDOT:PSS/SPCEs, and EPD rGO:PSS-PEDOT:PSS/SPCEs in $0.1 \mathrm{M}$ potassium ferricyanide, $\mathrm{K}_{3} \mathrm{Fe}(\mathrm{CN})_{6}$, over a potential range from $-0.5 \mathrm{~V}$ to $1.0 \mathrm{~V}$ at scan rates of $75,100,150,200$, and $250 \mathrm{mV} / \mathrm{s}$. Electrochemical parameters such as peak current $\left(I_{p}\right)$, effective surface area $\left(A_{e}\right)$, and separation of peak-to-peak potential $\left(\Delta \mathrm{E}_{\mathrm{p}}\right)$ were analyzed. The $I_{p}$ obtained from the measurement can be used to calculate the $A_{e}$ of electrodes using the Randles-Sevcik equation:

$$
\begin{gathered}
I_{p}=\left(2.69 \times 10^{5}\right) n^{3 / 2} D^{1 / 2} C A_{e} v^{1 / 2} \\
A_{e}=\frac{k}{\left[\left(2.69 \times 10^{5}\right) n^{\frac{3}{2}} D^{\frac{1}{2}} C\right]}
\end{gathered}
$$

\subsection{Scanning Electron Microscopy (SEM) of rGO:PSS-PEDOT:PSS/SPCES}

Surface morphologies of SPCEs, DC rGO:PSS-PEDOT:PSS/SPCEs, and EPD rGO:PSSPEDOT:PSS/SPCEs were observed using a scanning electron microscope (JSM-IT100, JEOL, JAPAN). The electrodes were coated with gold/palladium particles using the Quorum SC7620 Sputter Coater prior to imaging. SEM images were obtained with a power setting of $8.0 \mathrm{kV}$ and magnification of $300 \times$. The average diameter of rGO:PSS-PEDOT:PSS globules was determined using Image J software for $\mathrm{n}=15$.

\section{Results}

\subsection{Cyclic Voltammetry of $r G O: P S S-P E D O T: P S S / S P C E S$}

CV analysis was performed for bare SPCEs, EPD rGO:PSS-PEDOT:PSS/SPCEs, and DC rGO:PSS-PEDOT:PSS/SPCEs in $0.1 \mathrm{M} \mathrm{Fe}(\mathrm{CN})_{6^{4 / 3-}}$ to investigate the changes in $\mathrm{I}_{\mathrm{p}}, \mathrm{A}_{\mathrm{e}}$, and $\Delta \mathrm{E}_{\mathrm{p}}$. We determined the $\mathrm{I}_{\mathrm{p}}$ of EPD and DC rGO:PSS-PEDOT:PSS/SPCEs compared to bare SPCEs by analyzing the difference in anodic peak current $(\triangle \mathrm{I})$ between the bare and modified electrodes at a scan rate of $100 \mathrm{mV} / \mathrm{s}$. There was a noticeable increase in the $\mathrm{I}_{\mathrm{p}}$ of EPD and DC rGO:PSS-PEDOT:PSS/SPCEs compared to that of bare SPCEs ( $\mathrm{I}_{\mathrm{P}_{-} \text {Bare }}=0.247 \mathrm{~mA}$, $\mathrm{I}_{\mathrm{P}_{-} \mathrm{EPD}}=0.415 \mathrm{~mA}$, and $\mathrm{I}_{\mathrm{P}_{-} \mathrm{DC}}=1.045 \mathrm{~mA} ; \Delta \mathrm{IEPD}$-Bare $=$ $0.168 \mathrm{~mA}$, and $\triangle \mathrm{IDC}$-Bare $=0.798 \mathrm{~mA}$ ). The increase in $\mathrm{I}_{\mathrm{p}}$ suggests that rGO:PSS-PEDOT:PSS can facilitate accessibility of the $\mathrm{Fe}(\mathrm{CN})_{6}{ }^{4 / 3}$ - to the electrode surface by improving interfacial electron transfer, thus enhancing the $\mathrm{I}_{\mathrm{p}}$ signals. Incorporation of PEDOT:PSS to the rGO increased the electron transport owing to $\pi-\pi$ interactions between PEDOT:PSS and rGO. The availability of $\pi$-bonding aromatic rings increases and contributes to a larger number of $\pi-\pi$ overlaps, leading to more electron delocalization around the aromatic ring and thus improving the electrical conductivity of the modified electrodes [34-36]. Note that rGO:PSS is rGO layers stabilized in polystyrenesulfonate, 
which inhibits rGO stacking while promoting hydrophilicity of the nanomaterial, thus allowing rGO:PSS to be dissolved in aqueous solution [37].

In addition, Ae was also determined by applying the Randles-Sevcik equation. The Ae for bare SPCEs, EPD rGO:PSS-PEDOT:PSS/SPCEs, and DC rGO:PSS-PEDOT:PSS/SPCEs were $3.1 \mathrm{~mm}^{2}, 5.7$ $\mathrm{mm}^{2}$, and $6.7 \mathrm{~mm}^{2}$, respectively, showing a 2-fold increase for modified SPCEs compared to bare SPCEs. The increase in Ae of the EPD and DC rGO:PSS-PEDOT:PSS/SPCEs in comparison to the bare SPCEs could be attributed to the increase in surface-to volume ratio of the nanomaterials, as well as to increase in current density and charge transferability [21].

Further analyses of $\mathrm{CV}$ graphs compared the effect of deposition method on separation of peakto-peak potentials $\left(\Delta \mathrm{E}_{\mathrm{p}}=\mathrm{E}_{\mathrm{pa}}-\mathrm{E}_{\mathrm{pc}}\right)$, where lower $\Delta \mathrm{E}_{\mathrm{p}}$ implies an enhanced electrocatalytic ability of $\mathrm{Fe}(\mathrm{CN})^{4 / 3-}$ at the electrode-analyte interface. We analyzed the $\Delta \mathrm{E}_{\mathrm{p}}$ of EPD rGO:PSSPEDOT:PSS/SPCEs in comparison to DC rGO:PSS-PEDOT:PSS/SPCEs. The EPD rGO:PSSPEDOT:PSS/SPCEs showed a lower $\triangle \mathrm{E}_{\mathrm{p}}$ of $360 \mathrm{mV}, 1.4$ times lower than those of DC rGO:PSSPEDOT:PSS/SPCEs $\left(\triangle \mathrm{E}_{\mathrm{p}}=510 \mathrm{mV}\right)$. The lower $\Delta \mathrm{E}_{\mathrm{p}}$ suggests higher reversibility and faster electron transfer at the electrode-analyte interface; thus, the diffusion of analyte to the electrode surface is a dominant contributor to the peak potential [38]. The structure of PEDOT:PSS and rGO:PSS composites formed from the EPD process could potentially assist the electron transfer between electrode surface and analyte, making the nanocomposite suitable for ion-selective sensing.

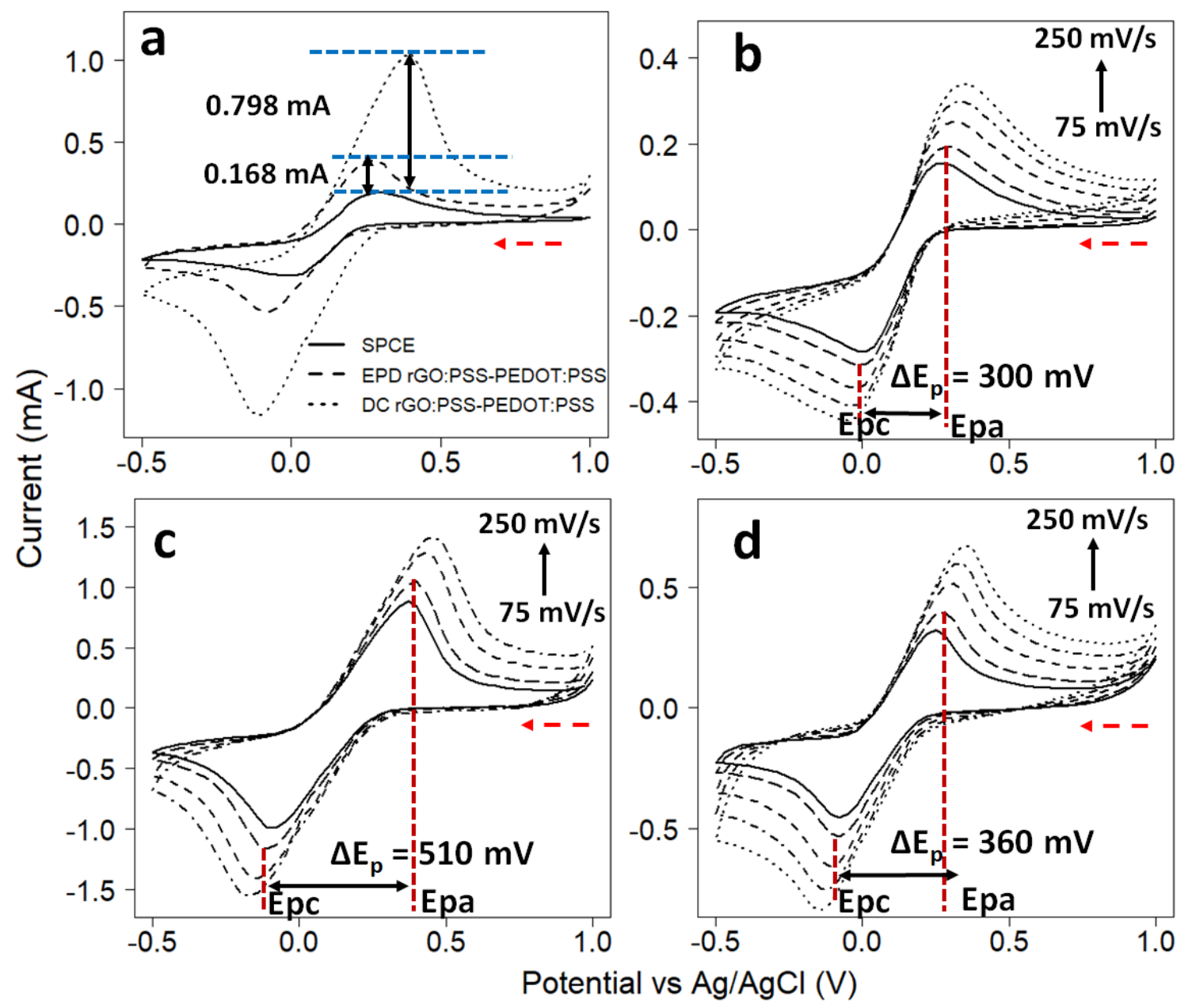

Figure 2. (a) Cyclic voltammetry (CV) measurements of bare SPCEs, EPD rGO:PSSPEDOT:PSS/SPCEs, and DC rGO:PSS-PEDOT:PSS/SPCEs at a scan rate of $100 \mathrm{mV} / \mathrm{s}$ in $0.1 \mathrm{M}$ $\mathrm{Fe}(\mathrm{CN}) 6^{4 / 3} \cdot \Delta \mathrm{I}_{\mathrm{p}}$ is shown. CV measurements of (b) SPCEs, (c) DC rGO:PSS-PEDOT:PSS/SPCEs, and (d) EPD rGO:PSS-PEDOT:PSS/SPCEs at scan rates of 75, 100, 150, 200, $250 \mathrm{mV} / \mathrm{s}$ in $0.1 \mathrm{M} \mathrm{Fe}(\mathrm{CN})^{4 / 3} . \Delta \mathrm{E}_{\mathrm{p}}$ is shown. 


\subsection{Surface Morphology of rGO:PSS-PEDOT:PSS/SPCES}

The surface morphology of SPCEs modified with EPD and DC of rGO:PSS-PEDOT:PSS was characterized under SEM. SEM images were taken after all electrodes were run through CV. The morphology could provide an insight on the effect of fluids on the nanocomposite after undergoing $\mathrm{CV}$, as $\mathrm{CV}$ is a consumptive process that can affect morphology of nanomaterial transducers. Figure 3 shows the surface morphology of bare SPCEs, PEDOT:PSS/SPCEs, DC rGO:PSSPEDOT:PSS/SPCEs, and EPD rGO:PSS-PEDOT:PSS/SPCEs. Bare SPCEs display a rough and flakylike morphology (Figure 3a), PEDOT:PSS/SPCEs appear smooth with some bumps, and DC rGO:PSSPEDOT:PSS/SPCEs resemble PEDOT:PSS/SPCEs (Figure 3b) but with the appearance of tiny grains on the electrode surface (Figure 3c). EPD rGO:PSS-PEDOT:PSS/SPCEs have the roughest surface and homogeneous globular structures can be seen to cover the electrode surface (Figure 3d). Detailed analysis using Image J software revealed that the globule diameters are in the range of $1.4-5.3 \mu \mathrm{m}$. In terms of electrode integrity in fluids, in this case after electrodes had undergone CV in $0.1 \mathrm{M}$ $\mathrm{Fe}(\mathrm{CN}) 6^{4 / 3-}$, cracks can be seen on DC PEDOT:PSS/SPCE surfaces (Figure 3b), whereas rGO:PSSPEDOT:PSS/SPCEs for both deposition methods of DC (Figure 3c) and EPD (Figure 3d) maintained their integrity. The results suggest that PEDOT:PSS alone can become brittle with prolonged measurement and storage in fluids. In PEDOT:PSS layers, PEDOT is surrounded by a PSS network that attracts a considerable amount of water, allowing transport and penetration of ions into the PEDOT layers. Ion transport, and subsequently electrochemical activity, become possible with such hydrated pathways. This, however, brings a challenge to maintain layer integrity since the layers are exposed to aqueous environment such as biological fluids for in-vivo applications and flowing water for environment applications [39]. In this regard, incorporation of rGO:PSS into PEDOT:PSS as a composite can improve the mechanical strength of rGO:PSS-PEDOT:PSS when electrodes undergo $\mathrm{CV}$ measurements. The results are consistent with previous studies that found that PEDOT:PSS/graphene nanocomposite [40] and PEDOT:PSS/carboxylic group functionalized graphene composite [41] are stable in aqueous media owing to the properties of graphene derivatives, which improve flexibility, enhance adhesion, and increase mechanical strength of the composite.

In addition, globular structures of rGO:PSS-PEDOT:PSS using EPD methods remained after undergoing $\mathrm{CV}$, indicating a robust and stable transducer in fluids. Besides morphological properties, the CV of EPD rGO:PSS-PEDOT:PSS have shown smaller $\triangle E_{p}$, which implies higher reversibility and faster electron transfer at the electrode-analyte interface. Tailoring the morphological and electrochemical properties of the EPD rGO:PSS-PEDOT:PSS/SPCEs, we can conclude that rGO:PSS-PEDOT:PSS via EPD can be a potential technique to fabricate a robust transducer for ion-selective sensing in biological and environmental fluids. Similar results were observed in previous work, where PEDOT:PSS drop-casting techniques produced smoother images when the electrodes were soaked in PBS; however, globular structures remained for electropolymerized PEDOT:PSS [31]. 


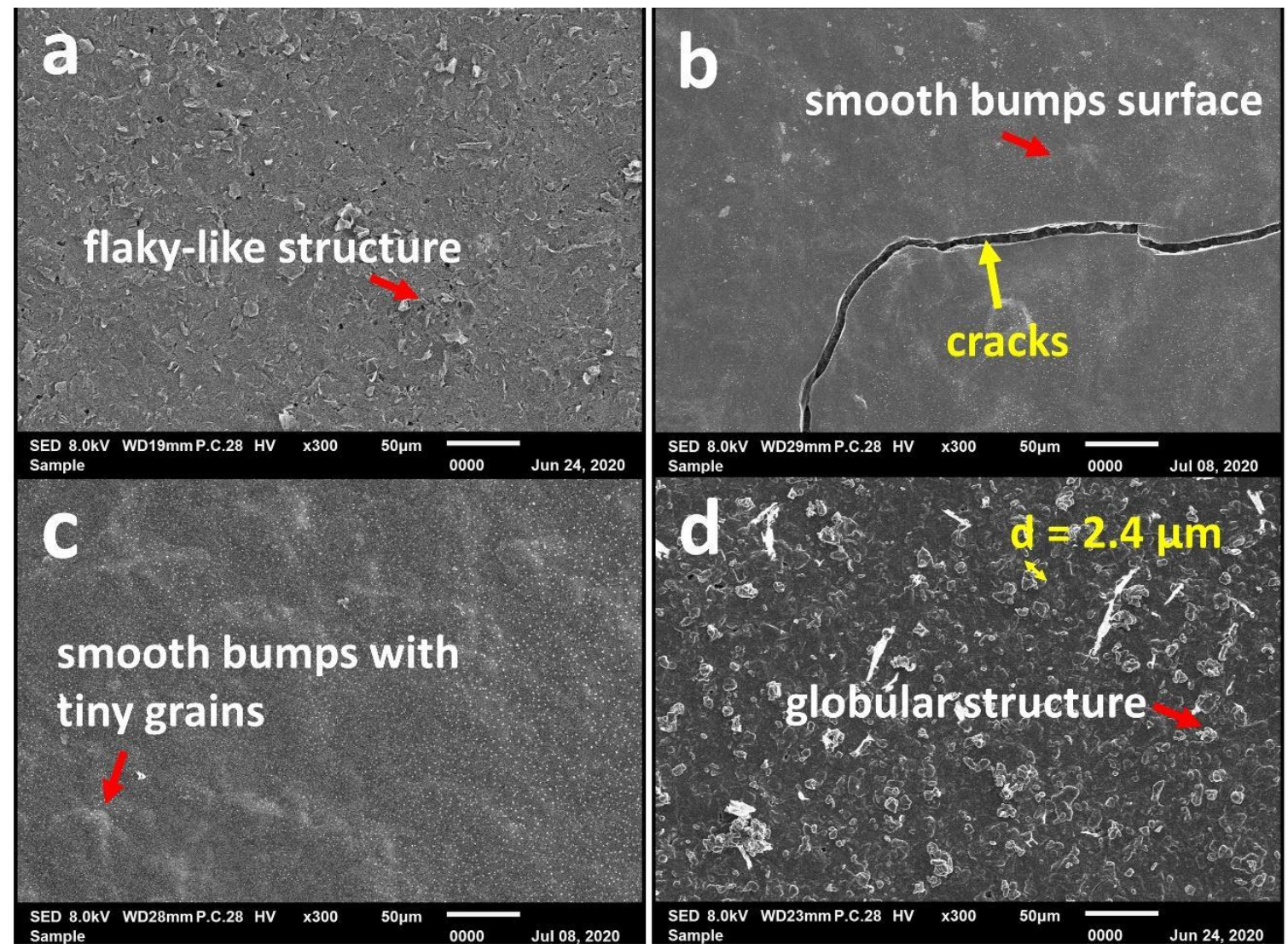

Figure 3. Surface morphology of (a) bare SPCEs, (b) PEDOT:PSS/SPCEs, (c) DC rGO:PSSPEDOT:PSS/SPCEs, and (d) EPD rGO:PSS-PEDOT:PSS/SPCEs. Bare SPCEs display a rough and flakylike morphology, PEDOT:PSS/SPCEs appear smooth with some bumps, and DC rGO:PSSPEDOT:PSS/SPCEs resemble PEDOT:PSS/SPCEs but with the appearance of tiny grains on the electrode surface. The roughest surface is EPD rGO:PSS-PEDOT:PSS/SPCEs where a homogeneous globular structure can be seen to cover the electrode surface. Detailed analysis using Image J software revealed that the globule diameters are in the range of $1.4-5.3 \mu \mathrm{m}$. In terms of electrode integrity in fluids, in this case after electrodes underwent $\mathrm{CV}$ in $0.1 \mathrm{M} \mathrm{Fe}(\mathrm{CN}) 6^{4 / 3-}$, cracks can be seen on DC PEDOT:PSS/SPCE surfaces, whereas rGO:PSS-PEDOT:PSS/SPCEs for both deposition methods of DC and EPD maintained their integrity. The results suggest that PEDOT:PSS alone can become brittle with prolonged measurement and storage in fluid. Incorporation of rGO:PSS into PEDOT:PSS as a composite improves the mechanical strength of rGO:PSS-PEDOT:PSS when electrodes undergo CV measurements.

\section{Conclusions}

We compared CV and surface morphology of rGO:PSS-PEDOT:PSS/SPCEs by two different techniques: electropolymerization deposition (EPD) and drop-casting (DC). Higher peak current $\left(\mathrm{I}_{\mathrm{p}}\right)$ was observed for nanocomposite-modified SPCEs with $\mathrm{I}_{\mathrm{P}_{-} \mathrm{EPD}}=0.415 \mathrm{~mA}$ and $\mathrm{I}_{\mathrm{p}_{-} \mathrm{DC}}=1.045 \mathrm{~mA}$, compared to bare SPCEs with $\mathrm{I}_{\text {p_Bare }}=0.247 \mathrm{~mA}$. The results indicate that the nanocomposite improves the conductivity of the working electrodes (WEs). Larger effective surface area $\left(\mathrm{Ae}_{\mathrm{e}}\right)$ was also observed for nanocomposite-modified SPCEs with $\mathrm{Ae}_{-} \mathrm{EPD}=5.7 \mathrm{~mm}^{2}$ and $\mathrm{Ae}_{-} \mathrm{DC}=6.7 \mathrm{~mm}^{2}$, showing a $\sim 2$-fold increase compared to bare SPCEs with Ae_Bare $=3.1 \mathrm{~mm}^{2}$. In addition, we analyzed the effect of deposition method on the electrochemical properties of the composite. Electropolymerized rGO:PSSPEDOT:PSS/SPCEs showed a smaller peak-to-peak potential separation $\left(\Delta \mathrm{E}_{\mathrm{p}}\right)$ of $360 \mathrm{mV}$ compared to the drop-cast rGO:PSS-PEDOT:PSS/SPCEs $(510 \mathrm{mV})$. The smaller $\Delta \mathrm{E}_{\mathrm{p}}$ of electropolymerized rGO:PSS-PEDOT:PSS suggests higher reversibility and faster electron transfer at the electrodeanalyte interface. SEM results showed that bare SPCEs display a rough and flaky-like morphology, 
PEDOT:PSS/SPCEs appear smooth with some bumps, and DC rGO:PSS-PEDOT:PSS/SPCEs resemble PEDOT:PSS/SPCEs but with appearance of tiny grains on the electrode surface. The roughest surface is EPD rGO:PSS-PEDOT:PSS/SPCEs, where a homogeneous globular structure can be seen to cover the electrode surface. Detailed analysis using Image J software revealed that the globule diameters are in the range of $1.4-5.3 \mu \mathrm{m}$. In terms of electrode integrity in fluids, in this case after electrodes underwent $\mathrm{CV}$ in $0.1 \mathrm{M} \mathrm{Fe}(\mathrm{CN}){ }^{4 / 3-}$, cracks can be seen on DC PEDOT:PSS/SPCE surfaces, whereas rGO:PSS-PEDOT:PSS/SPCEs for both deposition methods of DC and EPD maintained their integrity. The results suggest that PEDOT:PSS alone can become brittle with prolonged measurement and storage in fluids. Incorporation of rGO:PSS into PEDOT:PSS as a composite improves the mechanical strength of rGO:PSS-PEDOT:PSS when electrodes undergo CV measurements. Globular structures of rGO:PSS-PEDOT:PSS using EPD methods remained after undergoing CV. The results suggest that EPD serves as a potential method to fabricate a stable transducer for ion-selective sensing. Future work includes investigating the effect of ion-selective membranes on EPD rGO:PSSPEDOT:PSS/SPCEs, and testing the fabricated sensors for continuous monitoring in fluids. This study essentially offers a scalable platform to develop robust and stable ion-selective sensors, which are particularly useful for monitoring ion concentrations continuously in real-time for physiological and environmental applications.

Funding: This research was funded by nanoSkunkWorkX Sdn. Bhd.

Acknowledgments: The authors would like to thank Abdelmohsen Benoudjit, Piravin Raj Barthasarathy, and other members of Amani Research Group (ARG) for their support.

Conflicts of Interest: The authors declare no conflict of interest

\section{References}

1. Bobacka, J.; Ivaska, A.; Lewenstam, A. Potentiometric ion sensors. Chem. Rev. 2008, 108, 329-351.

2. Bakker, E.; Bühlmann, P.; Pretsch, E. Carrier-based ion-selective electrodes and bulk optodes. 1. General characteristics. Chem. Rev. 1997, 97, 3083-3132,

3. Bakker, E. Ion-Selective Electrodes, 3rd ed.; Elsevier Inc.: 2018.

4. Urbanowicz, M.; Jasiński, A.; Jasińska, M.; Drucis, K.; Ekman, M.; Szarmach, A.; Bocheńska, M. Simultaneous determination of $\mathrm{Na}^{+}, \mathrm{K}^{+}, \mathrm{Ca}^{2+}, \mathrm{Mg}^{2+}$, and $\mathrm{Cl}^{-}$in unstimulated and stimulated human saliva using all solid state multisensor platform. Electroanalysis 2017, 29, 1-8.

5. Piek, M.; Wojciechowska, A.; Fendrych, K.; Piech, R.; Paczosa-bator, B. A simple way to modify selectivity of sodium sensitive electrodes by using organic conductive crystals. Ionics (Kiel) 2018, 25, 2311-2321,

6. Tahirbegi, I.B.; Alvira, M.; Mir, M.; Samitier, J. Simple and fast method for fabrication of endoscopic implantable sensor arrays. Sensors 2014, 14, 11416-11426,

7. Catrall, R.W.; Freiser, H. Coated wire ion selective electrodes. Anal. Chem. 1971, 43, 1905-1906,

8. De Marco, R.; Veder, J.P.; Clarke, G.; Nelson, A.; Prince, K.; Pretsch, E.; Bakker, E. Evidence of a water layer in solid-contact polymeric ion sensors. Phys. Chem. Chem. Phys. 2008, 10, 73-76,

9. Huang, Y.; Wang, T.; Xu, Z.; Hughes, E.; Qian, F.; Lee, M.; Li, B. Real-time in situ monitoring of nitrogen dynamics in wastewater treatment processes using wireless, solid-state, and ion-selective membrane sensors. Environ. Sci. Technol. 2019, 53, 3140-3148,

10. Choosang, J.; Numnuam, A.; Thavarungkul, P.; Kanatharana, P.; Radu, T.; Ullah, S.; Radu, A. Simultaneous detection of ammonium and nitrate in environmental samples using on ion-selective electrode and comparison with portable colorimetric assays. Sensors 2018, 18, 3555-3562,

11. Athavale, R.; Dinkel, C.; Wehrli, B.; Bakker, E.; Crespo, G.A.; Brand, A. Robust solid-contact ion selective electrodes for high-resolution in situ measurements in fresh water systems. Environ. Sci. Technol. Lett. 2017, 4, 286-291.

12. Cánovas, R.; Sánchez, S.P.; Parrilla, M.; Cuartero, M.; Crespo, G.A. Cytotoxicity study of ionophore-based membranes: Toward on-body and in vivo ion sensing. ACS Sensors 2019, 4, 2524-2535.

13. Liu, J.; Li, F.; Wang, Y.; Pan, L.; Lin, P.; Zhang, B.; Fei, F. A sensitive and specific nanosensor for monitoring extracellular potassium levels in the brain. Nat. Nanotechnol. 2020, 15, 321-330, 
14. Song, Y.A.; Melik, R.; Rabie, A.N.; Ibrahim, A.M.; Moses, D.; Tan, A.; Lin, S.J. Electrochemical activation and inhibition of neuromuscular systems through modulation of ion concentrations with ion-selective membranes. Nat. Mater. 2011, 10, 980-986,

15. Zhao, L.; Jiang, Y.; Wei, H.; Jiang, Y.; Ma, W.; Zheng, W.; Mao, L. In vivo measurement of calcium ion with solid-state ion-selective electrode by using shelled hollow carbon nanospheres as transducing layer. Anal. Chem. 2019, 91, 4421-4428.

16. Sundramoorthy, A.K.; Premkumar, B.S.; Gunasekaran, S. Reduced graphene oxide-poly(3,4ethylenedioxythiophene) polystyrenesulfonate based dual-selective sensor for iron in different oxidation states. ACS Sensors 2016, 1, 151-157,

17. Hu, J.; Stein, A.; Bühlmann, P. Rational design of all-solid-state ion-selective electrodes and reference electrodes. Trends Anal. Chem. 2016, 76, 102-114,

18. Mendecki, L.; Mirica, K.A. Conductive metal-organic frameworks as ion-to-electron transducers in potentiometric sensors. ACS Appl. Mater. Interfaces 2018, 10, 19248-19257,

19. Criscuolo, F.; Taurino, I.; Stradolini, F.; Carrara, S.; de Micheli, G. Highly-stable Li+ion-selective electrodes based on noble metal nanostructured layers as solid-contacts. Anal. Chim. Acta 2018, 1027, 22-32,

20. Pirovano, P.; Dorrian, M.; Shinde, A.; Donohoe, A.; Brady, A.J.; Moyna, N.M.; McCaul, M. A wearable sensor for the detection of sodium and potassium in human sweat during exercise. Talanta 2020, 219, 121145.

21. Abd-wahab, F.; Farhana, H.; Guthoos, A.; Salim, W.W.A. Solid-state characterization of rGO-PEDOT : PSS transducing material for enzymatic sensing. Biosensors 2019, 9, 1-14,

22. Salim, W.W.A.W.; Zeitchek, M.A.; Hermann, A.C.; Ricco, A.J.; Tan, M.; Selch, F.; Porterfield, D.M. Multianalyte biochip (MAB) based on all-solid-state ion-selective electrodes (ASSISE) for physiological research. J. Vis. Exp. 2013, 74, 50020,

23. Han, T.; Mattinen, U.; Bobacka, J. Improving the sensitivity of solid-contact ion-selective electrodes by using coulometric signal transduction. ACS Sensors 2019, 4, 900-906/

24. He, Q.; Das, S.R.; Garland, N.T.; Jing, D.; Hondred, J.A.; Cargill, A.A.; Claussen, J.C. Enabling inkjet printed graphene for ion selective electrodes with postprint thermal annealing. ACS Appl. Mater. Interfaces 2017, 9, 12719-12727.

25. Jiang, H.; Yu, W.; Waimin, J.F.; Glassmaker, N.; Raghunathan, N.; Jiang, X.; Rahimi, R. Inkjet-printed solidstate potentiometric nitrate ion selective electrodes for agricultural application. IEEE Sensors 2019, 1-4.

26. Pol, R.; Moya, A.; Gabriel, G.; Gabriel, D.; Céspedes, F.; Baeza, M. Inkjet-printed sulfide-selective electrode. Anal. Chem. 2017, 89, 12231-12236.

27. Benoudjit, A.; Shohibuddin, I.U.S.; Salim, W.W.A.W. Comparative study of cyclic voltammetry and cycle stability of electropolymerized poly(3,4-ethylenedioxythiophene):poly(sodium 4-styrenesulfonate) on screen-printed electrodes in aqueous media. TEST Eng. Manag. 2020, 83, 1033-1040,

28. Lindfors, T.; Boeva, Z.A.; Latonen, R.M. Electrochemical synthesis of poly(3,4-ethylenedioxythiophene) in aqueous dispersion of high porosity reduced graphene oxide. RSC Adv. 2014, 4, 25279-25286.

29. Yang, T.; Yin, H.; Gao, L.H.; Wang, K.Z.; Yan, D. Recent advances in electrodes modified with ruthenium complexes for electrochemical and photoelectrochemical water oxidation. In Advances in Inorganic Chemistry, 1st ed.; van Eldik, R., Hubbard, C., Eds.; Academic Press: Hampshire Street, MA, USA, 2019, Volume 74, pp. 305-341.

30. Yang, X.; Kirsch, J.; Olsen, E.V.; Fergus, J.W.; Simonian, A.L. Anti-fouling PEDOT:PSS modification on glassy carbon electrodes for continuous monitoring of tricresyl phosphate. Sensors Actuators B Chem. 2013, 177, 659-667,

31. Benoudjit, A.; Bader, M.M.; Salim, W.W.A.W. Study of electropolymerized PEDOT : PSS transducers for application as electrochemical sensors in aqueous media. Sens. Bio Sensing Res. 2018, 17, 18-24.

32. Jiang, F.; Yao, Z.; Yue, R.; Du, Y.; Xu, J.; Yang, P.; Wang, C. Electrochemical fabrication of long-term stable Pt-loaded PEDOT/graphene composites for ethanol electrooxidation. Int. J. Hydrogen Energy 2012, 37, 14085-14093.

33. Foronda, J.R.F.; Cabrera, S.M.R.; Cumpas, D.L.; Villar, P.G.A.; Tan, J.L.; Tongol, B.J.V. Enhanced electrocatalytic activity of $\mathrm{Pt}$ particles supported on reduced graphene oxide/poly(3,4ethylenedioxythiophene) RGO/PEDOT composite towards ethanol oxidation. J. Chem. 2013, 2013, doi:10.1155/2013/501824. 
34. Cui, L.; Gao, J.; Xu, T.; Zhao, Y.; Qu, L. Polymer/graphene hybrids for advanced energy-conversion and storage materials. Chem. An Asian J. 2016, 11, 1151-1168.

35. Kertesz, M. Pancake bonding: An unusual pi-stacking interaction. Chem. A Eur. J. 2019, 25, 400-416.

36. Zhu, Y.; Murali, S.; Cai, W.; Li, X.; Suk, J.W.; Potts, J.R.; Ruoff, R.S. Graphene and graphene oxide: Synthesis, properties, and applications. Adv. Mater. 2010, 22, 3906-3924.

37. Chen, Y.L.; Chiang, J.Y.; Chou, H.T.; Fu, C.Y.; Chen, Y.C.; Lee, C.Y.; Chang, H.Y. Toxicity analysis of poly(sodium-4-styrenesulfonate) coated graphene on HMEC-1 cells under dynamic conditions mimicking blood flow. RSC Adv. 2017, 7, 51910-51918.

38. Graham, D.J. Standard Operating Procedures for Cyclic Voltammetry; USA, 2018.

39. Elmahmoudy, M.; Inal, S.; Charrier, A.; Uguz, I.; Malliaras, G.G.; Sanaur, S. Tailoring the electrochemical and mechanical properties of PEDOT:PSS films for bioelectronics. Wiley 2018, 1404, 11, doi:10.1002/mame.201600497.

40. Liu, D.; Rahman, M.M.; Ge, C.; Kim, J.; Lee, J.J. Highly stable and conductive PEDOT:PSS/graphene nanocomposites for biosensor applications in aqueous medium. New J. Chem. 2017, 41, 15458-15465.

41. Wang, Z.; Xu, J.; Yao, Y.; Zhang, L.; Wen, Y.; Song, H.; Zhu, D. Facile preparation of highly water-stable and flexible PEDOT:PSS organic/inorganic composite materials and their application in electrochemical sensors. Sensors Actuators B Chem. 2014, 196, 357-369.

Publisher's Note: MDPI stays neutral with regard to jurisdictional claims in published maps and institutional affiliations.

(C) 2020 by the authors. Licensee MDPI, Basel, Switzerland. This article is an open access article distributed under the terms and conditions of the Creative Commons Attribution (CC BY) license (http://creativecommons.org/licenses/by/4.0/). 